\title{
Peculiar velocity field in structure formation theories with cosmic strings
}

\author{
Carsten van de Bruck* \\ Institut für Astrophysik und Extraterrestrische Forschung Auf dem Hügel 71, 53121 Bonn, Germany
}

(Received 29 October 1997; published 30 March 1998)

\begin{abstract}
We investigate the peculiar velocity field due to long cosmic strings in several cosmological models and analyze the influence of a nonscaling behavior of the string network, which is expected in open cosmological models or models with a cosmological constant. It is shown that the deviation of the probability distribution of the peculiar velocity field from the normal distribution is only weak in all models. It is further argued that one cannot necessarily obtain the parameter $\beta=\Omega_{0}^{0.6} / b$ from density and velocity fields, where $\Omega_{0}$ is the density parameter and $b$ the linear biasing parameter, if cosmic strings are responsible for structure formation in the universe. An explanation for this finding is given. [S0556-2821(98)06708-3]

PACS number(s): 98.80.Cq, 11.27. $+\mathrm{d}, 98.65 .-\mathrm{r}$
\end{abstract}

\section{INTRODUCTION}

To understand the origin and the formation of structure in the universe is one of the most challenging problems in modern cosmology. There are two competitive theories which try to explain the origin of the seeds. The first one is inflation, in which the universe undergoes an epoch of fast (inflationary) expansion, triggered by a scalar field, called the inflaton. Quantum fluctuations in the inflaton field are stretched on superhorizon scales which are turned into matter fluctuations at later times. These matter fluctuations represent the seeds of the observed structure today [1]. In the other theory topological defects are responsible for structure formation. For example, cosmic strings might be produced in a phase transition in the very early universe. If they are heavy enough, they influence the cosmological fluid and could seed the structure in the universe (see [2,3] for reviews and references).

There are several ways to test these theories. For example, they make different predictions for the angular fluctuation spectrum of the cosmic microwave background anisotropy (CMBR) on small scales. The predicted spectra can then be compared with the data, for example of future projects such as the Microwave Anisotropy Probe (MAP) and PLANCK. Related to this is the comparison of the predicted matter power spectrum with the data. Cosmic strings could further be tested with "astrophysical' tests, such as the expected gravitational radiation background from strings, etc. It is interesting to note that all these tests gave consistent results for the string parameter $\mu$, the mass per length on the string $[2,3]$.

Another possibility for testing structure formation theories was proposed by [4]. The probability distribution of the peculiar velocity field should be different in inflationary models and models with topological defects such as cosmic strings. However, as emphasized by $[5,6]$, the probability distribution of the peculiar velocity field in cosmic string theories is Gaussian to high accuracy. This conclusion was based on the assumption that the string network reaches a scaling behavior.

\footnotetext{
*Email address: cvdb@astro.uni-bonn.de
}

It was shown by several authors that the scaling solution is only expected in the Einstein-de Sitter model [7,8]; in open models, in flat models with a cosmological constant, and in closed (loitering) models the behavior of the network is different from scaling. Further, the transition to the matter scaling behavior is much longer than previously estimated [9]. These possible sources of deviation from the string scaling solution should have interesting consequences. A first step was done in [10] and [11]. Whereas in [10] it was shown that only a drastic departure from scaling could solve the problems of structure formation with cosmic strings, [11] have shown that there is only weak dependence of the density parameter $\Omega_{0}$ in open and flat models with cosmological constant on the normalization of $G \mu$ from Cosmic Background Explorer (COBE) data. It might be, that the cosmic string scenario is successful also in the absence of the scaling behavior, i.e., that it works well in open models or in models with a cosmological constant.

In this paper we investigate the influence of cosmic strings on the peculiar velocity field. Earlier investigations of the peculiar velocity field concentrated on the spectrum of the field, i.e., its dependence on the length scale $L$, see, e.g., $[5,12,13]$. We are interested in the effects of a departure from the scaling behavior. We use an approximation, first introduced by [5] to calculate the effects of many strings.

The paper is organized as follows. In Sec. II we discuss the influence of cosmic strings on the peculiar velocity field. Our calculations of the string network are based on the calculations by [8]. Our results for the peculiar velocities are presented in Sec. III. In Sec. IV we argue that if cosmic strings seed the structure in the universe then the peculiar velocity field and the density field is correlated but one cannot obtain information on the parameter $\beta=\Omega_{0}^{0.6} / b$, where $\Omega_{0}$ is the density parameter and $b$ is a linear bias parameter. In Sec. $\mathrm{V}$ we summarize our results and give some conclusions. Throughout the paper we set $c=1$.

\section{THE PECULIAR VELOCITY FIELD DUE TO LONG COSMIC STRINGS}

The space-time of a straight cosmic string is similar to the Minkowski space-time, except for a deficit angle $\Delta \phi$, given by $[2,3]$ 


$$
\Delta \phi=8 \pi G \mu \gamma_{s} v_{s} .
$$

As a result, the matter gets a kick towards the plane swept out by the string $\left(v_{s}\right.$ is the string velocity and $\gamma_{s}$ is the Lorentz factor). The velocity kick due to a wiggly string is given by

$$
u_{s}=4 \pi G \mu \gamma_{s} v_{s} f=3.8(G \mu)_{6}\left(\gamma_{s} v_{s}\right) f \mathrm{~km} / \mathrm{s},
$$

with

$$
f=1+\frac{1}{2 v_{s}^{2} \gamma_{s}^{2}}\left(1-\frac{T}{\mu}\right) .
$$

The term $f$ corresponds to the small scale structure on the string, where $\mu$ is the effective mass per unit length on the string and $T$ is the string tension.

\section{A. The Zeldovich approximation}

To calculate the peculiar velocity field, we use the Zeldovich approximation, in which the physical coordinates of a particle are written by

$$
\mathbf{r}(\mathbf{x}, t)=a(t)[\mathbf{x}+\psi(\mathbf{x}, t)],
$$

where $a(t)$ is the scale factor, $\mathbf{x}$ is the comoving coordinate, and $\psi$ is the displacement vector due to inhomogeneities in the cosmic fluid, i.e., cosmic strings in our context [14]. The equation of motion is given by Newton's law

$$
\ddot{\mathbf{r}}=-\nabla_{\mathbf{r}} \boldsymbol{\Phi} .
$$

The gravitational field is connected with the matter distribution (Poisson equation), which can be obtained from linearizing Einstein's field equation:

$$
\nabla_{r}^{2} \boldsymbol{\Phi}=4 \pi G\left(\rho_{b}+\delta \rho\right)+\Lambda c^{2} .
$$

In this equation $\rho_{b}$ is the matter density, $\delta \rho$ the matter density fluctuation, and $\Lambda$ the cosmological constant. To first order one obtains

$$
\delta \equiv \frac{\rho-\rho_{b}}{\rho_{b}}=-\nabla_{\mathbf{x}} \cdot \psi(\mathbf{x}, t),
$$

where $\rho$ is the total matter density. This leads to

$$
\nabla_{\mathbf{r}}^{2} \psi=4 \pi G \rho_{b}\left(1-\nabla_{\mathbf{x}} \cdot \psi\right)+\Lambda c^{2} .
$$

Integration of this equation and substitution of

$$
\ddot{\mathbf{r}}=\frac{\ddot{a}}{a} \mathbf{r}+2 \dot{a} \dot{\psi}+a \ddot{\psi}
$$

and the second Friedmann equation

$$
\frac{\ddot{a}}{a}=-\frac{4 \pi G}{3} \rho_{b}+\frac{\Lambda c^{2}}{3}
$$

leads to the evolution equation for the displacement $\psi[14]$ :

$$
\ddot{\vec{\psi}}+2 \frac{\dot{a}}{a} \dot{\vec{\psi}}-4 \pi G \rho_{b} \vec{\psi}=0 .
$$

For our purposes we have to calculate the peculiar velocity field, which can be obtained from Eq. (2.4):

$$
\mathbf{v}_{\mathrm{pec}}=a \dot{\psi} .
$$

The effect of the cosmological constant on the evolution of a density perturbation is only due to the effect of $\Lambda$ on the evolution of the scale factor $a$.

\section{B. The influence of cosmic strings}

We use an analytical approximation (the so called multiple impulse approximation), first introduced by Vachaspati [5], which also was successfully applied to calculate the CMBR anisotropies [15]. We divide the time interval from $t_{e q}$ (at which structures start to form) to $t_{0}$ in $N$ steps with $t_{i+1}=2 t_{i}$. Between $t_{i}$ and $t_{i+1}$ the strings intercommute, form loops, etc., so that (approximately) at $t_{i+1}$ the "new ordered state" of the network is uncorrelated with the "old state" at $t_{i}$. Again, at this time the network influences the matter within the horizon (due to scalar field radiation scales larger than the horizon are not affected). This is not true in vacuum dominated epochs. In this case the velocity of the strings decreases and therefore the probability of string interaction decreases. This means that the new state is correlated with the old one. However, in such epochs the number of strings within the Hubble horizon decreases rapidly and therefore our results are not changed significantly (see below).

At $t_{1}=t_{e q}$ each string within the horizon gives the matter a kick in the direction of the surface swapped out by the string:

$$
\mathbf{v}_{1, i}=u_{s} \mathbf{k}_{i, 1},
$$

where $\mathbf{k}_{i, 1}$ is a (random) unit vector in direction of the string $i$. The resulting peculiar velocity from all strings at $t_{1}$ is

$$
\mathbf{v}_{1}=\sum_{i=1}^{n_{g, 1}} u_{s} \mathbf{k}_{i, 1} \text {. }
$$

The sum is now taken over the number $n_{g, 1}$ of all strings within the horizon at $t_{1}$. This peculiar velocity field grows between $t_{1}$ and $t_{2}$ by a factor $\mathcal{A}\left(t_{1}, t_{2}\right)$ via Eqs. (2.11) and (2.12):

$$
\mathcal{A}\left(t_{i}, t_{f}\right)=\frac{a\left(t_{f}\right)\left|\dot{\psi}\left(t_{f}\right)\right|}{a\left(t_{i}\right)\left|\dot{\psi}\left(t_{i}\right)\right|} .
$$

At the time $t_{2}$ the peculiar velocity field is given by

$$
\mathbf{v}_{2}=\mathcal{A}\left(t_{1}, t_{2}\right) \mathbf{v}_{1}+u_{s} \sum_{j=1}^{n_{g, 2}} \mathbf{k}_{2, j}=u_{s}\left[\sum_{i=1}^{2} \sum_{j=1}^{n_{g, i}} \mathcal{A}\left(t_{2}, t_{i}\right) \mathbf{k}_{i, j}\right] .
$$

Here we have used that $\mathcal{A}\left(t_{i}, t_{j}\right) \mathcal{A}\left(t_{j}, t_{l}\right)=\mathcal{A}\left(t_{i}, t_{l}\right)$ and that the velocity of the strings is the same in every epoch. For our purposes this is a good approximation, because when the strings slow down the number of strings within the horizon also decreases.

Iteration leads to the peculiar velocity field at the present time on a scale $L$ :

$$
\mathbf{v}_{0}(L)=u_{s}\left[\sum_{i=1}^{N_{L}} \sum_{j=1}^{n_{g, i}} \mathcal{A}\left(t_{0}, t_{i}\right) \mathbf{k}_{i, j}\right] .
$$


TABLE I. The four representative cosmological models. $K$ is the curvature parameter, $\Omega_{0}$ is the matter density parameter, $\lambda_{0}$ is the cosmological term, $H_{0}$ is the Hubble parameter (in $\left.\mathrm{km} \mathrm{s}^{-1} \mathrm{Mpc}^{-1}\right) . N_{e q}$ is the number of Hubble steps between $t_{e q}$ and $t_{0}$.

\begin{tabular}{crlllc}
\hline \hline Model & $K$ & $\Omega_{0}$ & $\lambda_{0}$ & $\mathrm{H}_{0}$ & $N_{e q}$ \\
\hline 1 & +1 & 0.014 & 1.08 & 90 & 13 \\
2 & 0 & 1.0 & 0.0 & 60 & 20 \\
3 & -1 & 0.1 & 0.0 & 60 & 14 \\
4 & 0 & 0.1 & 0.9 & 60 & 15 \\
\hline \hline
\end{tabular}

In this equation $N_{L}$ is the number of Hubble time steps during which a volume of comoving size $L^{3}$ experiences string impulses, $n_{g, i}$ is the number of strings within the horizon at the time $t_{i}$. We assume that the vectors $\mathbf{k}_{i, j}$ are random, that is,

$$
\left\langle\mathbf{k}_{i, j} \cdot \mathbf{k}_{l, m}\right\rangle=\delta_{i l} \delta_{j m}
$$

From these equations we calculate the rms velocity numerically on a scale $L\left(t_{e q}\right)$. On scales smaller than $L\left(t_{e q}\right)$ the peculiar velocity field depends only weakly on $L$ whereas on scales larger than $L\left(t_{e q}\right)$ the predicted velocity field scales as $L^{-1}[12]$.

\section{Network parameter}

We use the calculation from [8] for the statistical properties of the string network. We set the number of strings within the horizon $H^{-1}$ by

$$
n_{s}=1+(\xi \cdot H)^{-1}
$$

where $\xi$ is the characteristic length scale of the string network, defined by

$$
\rho_{\infty}=\frac{\mu}{\xi^{2}},
$$

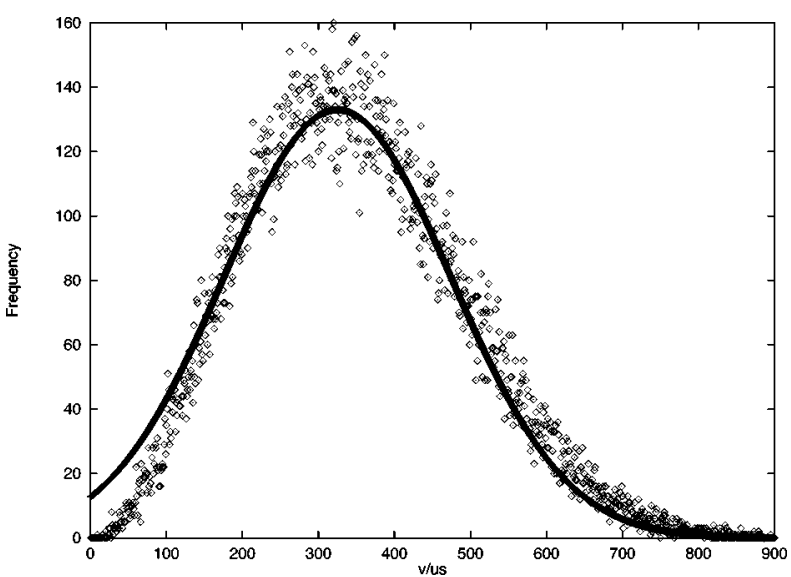

FIG. 1. The probability distribution of the peculiar velocity field at a scale corresponding to $t_{e q}$ in the EdS model with ideal scaling. The solid curve is the normal distribution. The mean value of $u_{\mathrm{pec}} / u_{s}$ is given by 325 and the standard deviation by 150 .

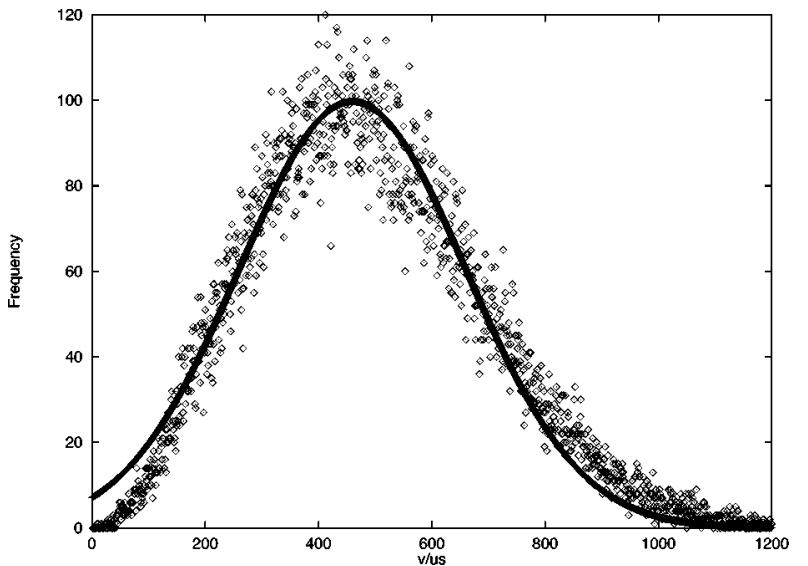

FIG. 2. The probability distribution of the peculiar velocity field at a scale corresponding to $t_{e q}$ in the EdS model with nonideal scaling. The solid curve is the normal distribution. The mean value of $v_{\text {pec }} / u_{s}$ is given by 460 and the standard deviation by 200 .

where $\rho_{\infty}$ is the density of the long strings. In the radiation dominated epoch $n_{s}$ is about 10, in the (late) matter dominated epoch (with scaling) this number is about 3 . In more general cosmological models this number is a function of time [8]. Later we will discuss the influence of the ansatz (2.19).

\section{RESULTS}

We calculate the peculiar velocity field for four representative models, shown in Table I. For the Einstein-de Sitter (EdS) model we discuss the influence of the long transition between the radiation and matter scaling solution [9]. As a test, we include the case for an ideal scaling in the EdS model. The propability distributions of the peculiar velocity fields at a scale $L\left(t_{e q}\right)$ for the models are shown in Figs. $1-5$. Each plot was obtained after 50000 realizations. For an exact scaling behavior in the EdS model this distribution was shown to be Gaussian $[5,6]$. We obtain the same result (see Fig. 1). In the case of the long transition between the radiation and matter scaling behavior the distribution remains nearly Gaussian. However, the distribution becomes broader

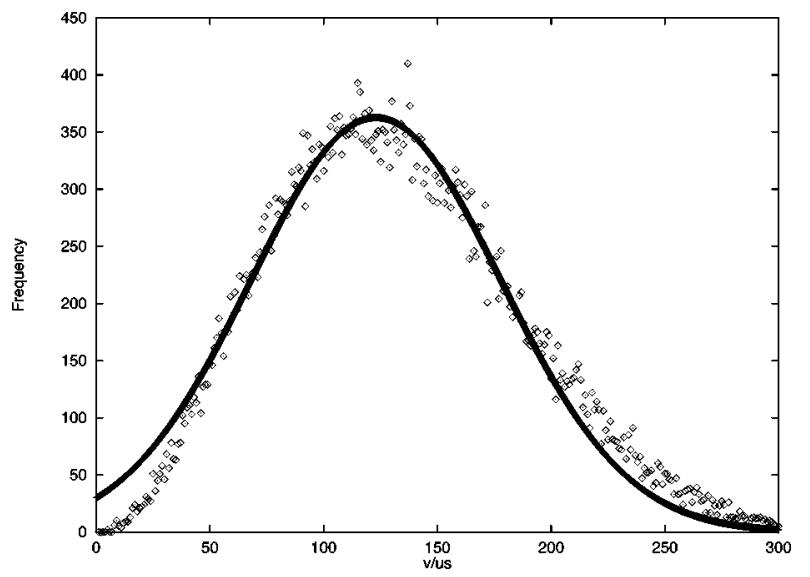

FIG. 3. The probability distribution of the peculiar velocity field at a scale corresponding to $t_{e q}$ in the closed model. The solid curve is the normal distribution. The mean value of $v_{\mathrm{pec}} / u_{s}$ is given by 123 and the standard deviation by 55 . 


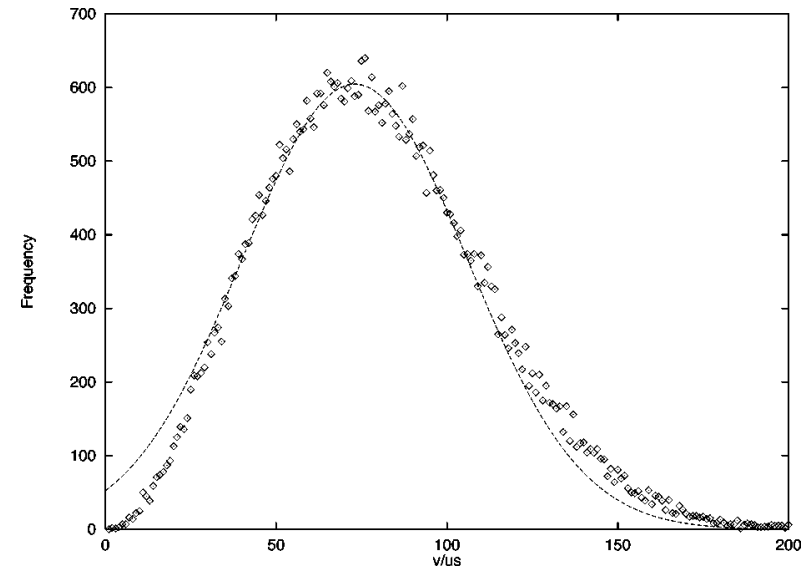

FIG. 4. The probability distribution of the peculiar velocity field at a scale corresponding to $t_{e q}$ in the flat model with cosmological constant. The solid curve is the normal distribution. The mean value of $v_{\text {pec }} / u_{s}$ is given by 73 and the standard deviation by 33 .

and the peculiar velocity increases (Fig. 2). The Gaussian character of the probability distribution can be found in the other models, too. There is only a slight deviation at large and small velocities.

In the models, we obtain a peculiar velocity at a scale corresponding to $L\left(t_{e q}\right)$ given by [the length scales are calculated with $\left.H_{0}=100 \mathrm{~km} /(\mathrm{s} \mathrm{Mpc})\right]$

$$
\begin{gathered}
v_{\mathrm{pec}}\left(L_{e q} \approx 70 \mathrm{Mpc}\right)_{\mathrm{closed}}=(460 \pm 200)(G \mu)_{6}\left(\gamma_{s} v_{s}\right) f \begin{array}{r}
\mathrm{km} / \mathrm{s}, \\
(3.1)
\end{array} \\
v_{\mathrm{pec}}\left(L_{e q} \approx 1 \mathrm{Mpc}\right)_{\mathrm{EdS}, \mathrm{ni}}=(1740 \pm 760)(G \mu)_{6}\left(\gamma_{s} v_{s}\right) f \begin{array}{r}
\mathrm{km} / \mathrm{s}, \\
(3.2)
\end{array} \\
v_{\mathrm{pec}}\left(L_{e q} \approx 1 \mathrm{Mpc}\right)_{\mathrm{EdS}, \mathrm{id}}=(1240 \pm 570)(G \mu)_{6}\left(\gamma_{s} v_{s}\right) f \begin{array}{r}
\mathrm{km} / \mathrm{s}, \\
(3.3)
\end{array} \\
v_{\mathrm{pec}}\left(L_{e q} \approx 10 \mathrm{Mpc}\right)_{\lambda, \text { flat }}=(280 \pm 120)(G \mu)_{6}\left(\gamma_{s} v_{s}\right) f \begin{array}{r}
\mathrm{km} / \mathrm{s}, \\
(3.4)
\end{array} \\
v_{\mathrm{pec}}\left(L_{e q} \approx 10 \mathrm{Mpc}\right)_{\mathrm{open}}=(80 \pm 35)(G \mu)_{6}\left(\gamma_{s} v_{s}\right) f \mathrm{~km} / \mathrm{s} .
\end{gathered}
$$

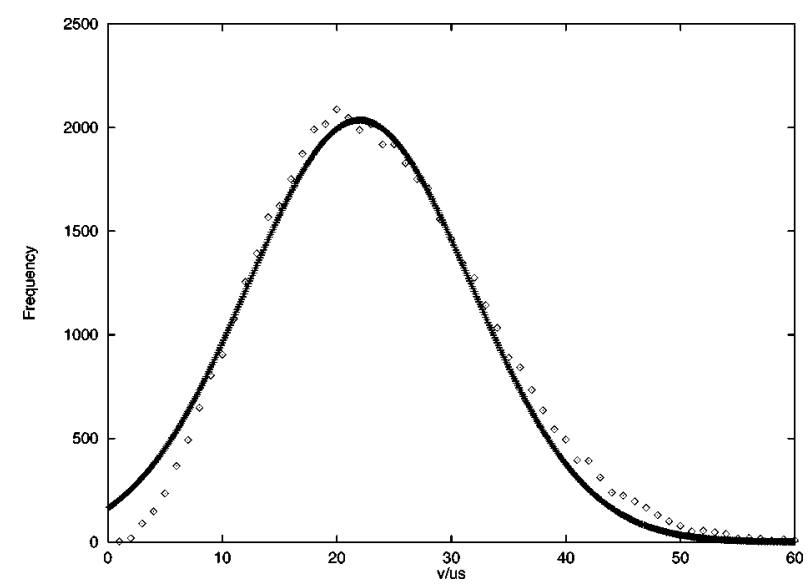

FIG. 5. The probability distribution of the peculiar velocity field at a scale corresponding to $t_{e q}$ in the open model. The solid curve is the normal distribution. The mean value of $v_{\text {pec }} / u_{s}$ is given by 22 and the standard deviation by 9.8 .
The length scale corresponding to the time $t_{e q}$ is set to be $0.1 H\left(t_{e q}\right)^{-1}[5]$ :

$$
L_{e q} \approx 1.1 \frac{1}{\Omega_{0}} h_{0}^{-2} \mathrm{Mpc} .
$$

Note, that in [5] the length scale was set to be $0.7 t_{e q}$. Therefore, we assume a somewhat pessimistic view when strings could significantly influence the volume at $t_{e q}$. In our picture, the volume must be within the typical length scale between all strings. However, the volume is influenced by the strings outside the volume and therefore we somewhat underestimate the peculiar velocities. However, this can be taken into account with including a parameter $\zeta$, which modifies our ansatz (2.19) [see below, Eq. (3.8)].

Within this length the peculiar velocity remains nearly constant, because a smaller length corresponds to times $t$ $<t_{e q}$ in which perturbation grow only weakly. This would imply that for the closed model we would expect nearly constant bulk flows on scales smaller than $70 \mathrm{Mpc}$, which is indeed observed. The situation in the other models is not so clear, because for scales larger than $L_{e q}$ the velocity decreases as $L$ increases $\left(v \propto L^{-1}\right)$.

It is interesting to note that in all models the standard deviation is related to the mean value by

$$
\sigma \approx 0.45 v_{\text {mean }}
$$

For our calculations we have used the ansatz (2.19) for the number of strings within the horizon. Although this should be a good approximation we could set $n_{s}=\zeta[1$ $\left.+(\xi \cdot H)^{-1}\right]$. The frequency distribution remains Gaussian, however, now the rms velocity and the standard deviation is given by

$$
v_{\text {pec }, \zeta}=\sqrt{\zeta} v_{\text {pec }, \zeta=1}
$$

and

$$
\sigma_{\zeta}=\sqrt{\zeta} \sigma_{\zeta=1}
$$

Here, $v_{\text {pec }, \zeta=1}$ and $\sigma_{\zeta=1}$ is given by Eqs. (3.1)-(3.5) for the cosmological models. The peculiar velocities therefore depend on the parameter

$$
\alpha=\sqrt{\zeta} \mu_{6}\left(v_{s} \gamma_{s}\right) f
$$

To conclude, the fluctuation of the number of strings does not change the shape of the probability distribution of the peculiar velocities and the amplitude depends on the same set of parameters (3.10) as in the case for an ideal scaling behavior of the string network. However, the effective number of strings and the maximum length on which coherent bulk flows are expected, depends on the cosmological parameters $\Omega_{m, 0}, \lambda_{0}$, and $H_{0}$.

\section{MATTER DISTRIBUTION, BULK FLOWS, AND BIASING}

The results presented in the last section imply that the parameter $\beta=\Omega_{m, 0}^{0.6} / b$ could not be obtained from velocitydensity reconstruction methods such as POTENT. To see this, we remember that the fundamental equation, on which 
these kinds of reconstruction methods are based, is given by $[16,17]$

$$
\nabla \cdot \mathbf{v}=-\beta H \delta
$$

Here, $\delta$ is the density fluctuation. On the other side, the continuity equation holds:

$$
\nabla \cdot \mathbf{v}=-\dot{\delta}
$$

In fact, in linear approximation Eq. (4.1) can be obtained from Eq. (4.2). The important point is that if the ratio $\delta / \delta$ is independent of space, an arbitrary application of Eq. (4.1) can lead to an underestimation or overestimation of $\beta$ if one applies Eq. (4.1) arbitrarily to the data. This was shown by [18] in the context of the explosion scenario. To demonstrate this point we repeat their short analytical example.

Let us consider an empty universe with $\Omega_{0}=0$, filled with massless particles. At some time $t_{i}$, the matter gets a kick due to a cosmic string (in the paper by Babul et al., the case of the explosion scenario was considered, but in the case of cosmic strings the analysis is identical). The linear Euler equation reads

$$
\frac{\partial \mathbf{v}}{\partial t}+2 H(t) \mathbf{v}=\mathbf{v}_{\text {string }} \delta\left(T-t_{i}\right)
$$

The density contrast evolves according to

$$
\frac{\partial^{2} \delta}{\partial t^{2}}+2 H(t) \frac{\partial \delta}{\partial t}=0
$$

This equation can be solved with the boundary conditions at the time $t_{i}$, which are $\delta\left(x, t_{i}\right)=0$ and $\dot{\delta}\left(x, t_{i}\right)=\zeta\left(x, t_{i}\right)$ $=-\nabla_{x} \cdot \mathbf{v}_{\text {string }}\left(\mathbf{x}, t_{i}\right)$. The solution of the equation (4.4) is given by $\left[a\left(t_{i}\right)=1\right]$

$$
\begin{aligned}
\delta(\mathbf{x}, t) & =\zeta\left(\mathbf{x}, t_{i}\right) t_{i}[a(t)-1] / a(t), \\
t \dot{\delta}(\mathbf{x}, t) & =\zeta\left(\mathbf{x}, t_{i}\right) t_{i} / a(t)
\end{aligned}
$$

One can see, that the ratio $\dot{\delta} / \delta$ is independent of space. We can use the continuity equation (4.2) and Eq. (4.1) to get

$$
\beta_{\mathrm{eff}}=\frac{1}{a(t)-1}
$$

Although the true value is zero, an observer, applying Eq. (4.1) to the data, will get a value that is different from the true one. Only at late times $\beta_{\text {eff }}$ will approach the value $\beta$ $=0$.

We expect similar results for the models in Table I. The cosmological model changes the time dependence of $\delta$ and $\dot{\delta}$, but in general, if there were only one velocity kick on the matter, the ratio $\dot{\delta} / \delta$ would be independent of space.

We have seen, that in cosmic string models of structure formation the observed peculiar velocity field is a vector sum of many contributions of strings. We write the general solutions of $\dot{\delta}$ and $\delta$, which arise from one kick as

$$
\delta_{i, j}\left(\mathbf{x}, t_{i}\right)=\zeta\left(\mathbf{x}, t_{i}\right)_{i, j} \mathcal{B}\left(t, t_{i}\right)
$$

$$
\dot{\delta}_{i, j}\left(\mathbf{x}, t_{i}\right)=\zeta\left(\mathbf{x}, t_{i}\right)_{i, j} \mathcal{C}\left(t, t_{i}\right) .
$$

The fields $\dot{\delta}$ and $\delta$, which arise from all strings in the past are given by

$$
\begin{aligned}
& \dot{\delta}(\mathbf{x}, t)=\sum_{i=1}^{N} \sum_{j=1}^{n_{g, i}} \dot{\delta}_{i, j}(\mathbf{x}, l), \\
& \delta(\mathbf{x}, t)=\sum_{i=1}^{N} \sum_{j=1}^{n_{g, i}} \delta_{i, j}(\mathbf{x}, t) .
\end{aligned}
$$

If we now replace $\zeta_{i, j}$ by $\delta_{i, j} / \mathcal{B}$, we can write

$$
\dot{\delta}=\sum_{i=1}^{N} \sum_{j=1}^{n_{g, i}} \delta_{i, j}\left(\mathbf{x}, t_{i}\right) \frac{\mathcal{C}\left(t, t_{i}\right)}{\mathcal{B}\left(t, t_{i}\right)} .
$$

If we compare this with Eq. (4.8) we see that there is a relation between the velocity field and the density field, but not of the same form as in Eq. (4.1). For cosmic strings we could write

$$
\nabla \cdot \vec{v}=-\sum_{i=1}^{N} \sum_{j=1}^{n_{g, i}} \delta_{i, j}\left(\mathbf{x}, t_{i}\right) \frac{\mathcal{C}\left(t, t_{i}\right)}{\mathcal{B}\left(t, t_{i}\right)} .
$$

If we compare Eq. (4.13) with Eq. (4.1), we find that

$$
\beta_{\mathrm{eff}}=\frac{1}{H_{0}} \frac{\Sigma_{i, j} \delta_{i, j} \mathcal{C}_{i, j} / \mathcal{B}_{i, j}}{\Sigma_{i, j} \delta_{i, j}},
$$

where $\mathcal{B}$ and $\mathcal{C}$ are the solutions of the perturbation equations. This equation can be interpreted as follows: each kick gives an effective $\beta_{\text {eff }, i, j}=\mathcal{C}_{i, j} / \mathcal{B}_{i, j} H_{0}$. Therefore, Eq. (4.14) is the weighted mean of the $\beta_{\mathrm{eff}, i, j}$ :

$$
\beta_{\mathrm{eff}}=\frac{\Sigma_{i, j} \delta_{i, j} \beta_{\mathrm{eff}, i, j}}{\sum_{i, j} \delta_{i, j}} .
$$

This represents a measure of the departure of an exact relation between the velocity and density field. Its value will depend on the length scale and on $\Omega_{0}$ and $\lambda_{0}$.

\section{DISCUSSION AND CONCLUSIONS}

In this paper we have considered the properties of the peculiar velocity field of galaxies in structure formation theories with cosmic strings. We considered the fact that the string network might not have developed a scaling behavior (as is the case in open models or models with a cosmological constant) and showed that the probability distribution of the peculiar velocities is nearly Gaussian. The rms peculiar velocity depends on the (effective) number of strings within the horizon and on the string parameter $G \mu$. The length, within the peculiar velocity is nearly independent of the scale, depends on the cosmological parameter $\Omega_{0}, \lambda_{0}$, and $H_{0}$.

Open models have more problems with the amplitude of the peculiar velocity field. Only an unplausible high value of $f$ could solve the problems $(f \approx 5)$. The situation might be better in flat models with a cosmological constant. However, on scales larger than $L_{e q}$ the peculiar velocity drops with $L^{-1}$, that is on a scale of about $60 \mathrm{Mpc}$ we expect in the model four peculiar velocities of $50-100 \mathrm{~km} / \mathrm{s}$. This is not in agreement with the observed value of $350-450 \mathrm{~km} / \mathrm{s}$. The 
situation is very good in the closed model. On scales smaller than $L_{e q}$ the velocity increases only weakly as $L$ decreases $[5,12,13]$ and remains nearly constant at $460 \mathrm{~km} / \mathrm{s}$ (for $G \mu$ $=10^{-6}$ and $\left.f \gamma_{s} v_{s} \approx 1\right)$ up to scales of about $200 \mathrm{Mpc}$. Note that these conclusions depend on the value of $G \mu$.

The results imply that if cosmic strings seed the structure in the universe, one cannot necessarily obtain the density parameter from the data. Comparison of density fields and velocity fields lead to an effective value, which is a measure of the deviation of an exact relation between the velocity and density field. Further work should be done on structure formation with cosmic strings in order to investigate the effects on a nonscaling behavior of the cosmic string network.

\section{ACKNOWLEDGMENTS}

I thank Matthias Soika and Harald Giersche for discussions. This work was supported by the Deutsche Forschungsgemeinschaft (DFG).
[1] P. J. Steinhardt, in Particle and Nuclear Astrophysics and Cosmology in the Next Millennium, edited by E. W. Kolb and R. D. Peccei (World Scientific, Singapore, 1995).

[2] A. Vilenkin and E. P. S. Shellard, Cosmic Strings and Other Topological Defects (Cambridge University Press, Cambridge, England, 1994).

[3] M. Hindmarsh and T. W. B. Kibble, Rep. Prog. Phys. 58, 477 (1995).

[4] L. Kofman, E. Bertschinger, J. M. Gelb, A. Nusser, and A. Dekel, Astrophys. J. 420, 44 (1994).

[5] T. Vachaspati, Phys. Lett. B 282, 305 (1992).

[6] R. Moessner, Mon. Not. R. Astron. Soc. 277, 927 (1995).

[7] C. J. A. P. Martins, Phys. Rev. D 55, 5208 (1997).

[8] C. van de Bruck, Phys. Rev. D 57, 1306 (1998).

[9] C. J. A. P. Martins and E. P. S. Shellard, Phys. Rev. D 54, 2535 (1996).
[10] A. Albrecht, R. A. Battye, and J. Robinson, Phys. Rev. Lett. 79, 4736 (1997).

[11] P. Avelino, R. R. Caldwell, and C. J. A. P. Martins, Phys. Rev. D 56, 4568 (1997).

[12] T. Vachaspati and L. Perivolaropolous, Astrophys. J. 123, 456 (1994).

[13] T. Hara, P. Mähönen, and S. Miyoshi, Astrophys. J. 415, 445 (1993).

[14] Y. B. Zeldovich, Astron. Astrophys. 5, 84 (1970).

[15] L. Perivolaropoulos, Phys. Lett. B 298, 305 (1993).

[16] P. J. E. Peebles, The Large Scale Structure of the Universe (Princeton University Press, Princeton, 1980).

[17] A. Dekel, Annu. Rev. Astron. Astrophys. 32, 371 (1994).

[18] A. Babul, D. H. Weinberg, A. Dekel, and J. P. Ostriker, Astrophys. J. 427, 1 (1994). 